American Journal of Applied Sciences 7 (3): 349-351, 2010

ISSN 1546-9239

(C) 2010Science Publications

\title{
Myocardial-Infraction Based on Intelligent Techniques
}

\author{
Ibrahim Abdulrab Ahmed \\ Hodeidah University, Hodiedah, Republic of Yemen
}

\begin{abstract}
Problem statement: Heart disease is the leading cause of death in the world. One of the most common types of heart diseases is Myocardial-Infraction. The ability to automatically identify Myocardial-Infraction from cardiac profiles test is important for clinical diagnosis and treatment. Approach: The aim of this study is to see whether the heart is healthy or not, in another words to verify the presence or the absence of Myocardial-Infraction using selected classification methods (neural networks and support vector machines) and to compare the performance of these used methods. Input layer, consists of input features (measurement of labs. Testing for CK, GOT, LDH, Tr), hidden layer of 2 neurons, output layer of one neuron activated by logistic function, which determine if heart is healthy or not. Result: The classification showed 97\% for SVM-nonlinear classifier using radial function, 57\% for SVM-linear classifier and 90\% for Neural Networks (Back Propagation). Conclusion: The result strongly suggested that nonlinear SVMs can aid in the diagnosis of Myocardial-infraction.
\end{abstract}

Key words: Support vector machines, artificial neural networks

\section{INTRODUCTION}

The studies and researches have so far concentrated on the human being, as he is the base and essence for life continuation. And the necessary facilities for medical diagnosis have become at hand. For as we know if there is precise diagnosis then the physician can give appropriate medical prescription or at least prevent spreading of the disease. Using the neural networks, fuzzy system, genetic algorithms, machine learning techniques and SVM in medical diagnosis is considered one of the main interests of the researches nowadays. The techniques used in this study are SVMs and NNs. Recently SVMs have been used in a range of problems including bioinformatics (Yu et al., 2003), text categorization (Joachims and Chemintz, 1998), classification and pattern recognition (Pontil and Verri, 1998), whereas Neural Networks (NNs) learning methods provide a robust approach to approximating real-valued, discrete-valued and vector-valued target functions. For certain types of problems such as learning to interpret complex real-world sensor data, Neural Networks are among the most effective learning methods currently known (Mitchell, 1997).

The objective of this research is to diagnose myocardial-infraction through the use of neural networks and support vector machines.

Experiments design: We have used SVM-light package for Joachim and self-made code for back- propagation method. The datasets of Cardiac Profiles test (CK, GOT, LDH, TROPINON) used in this study were obtained for the patients from the Cardiac Clinic archive in Al_amal Hospital through November 2006May 2007, since: Creation Kines (CK) is an enzyme released from skeletal muscle. Creation Kines (CK$\mathrm{MB}$ ) the isoenzyme which is released from heart cells and specific indicator of cardiac muscle damage especially Myocardial Infraction (MI)-TROPINON is one of a complex of proteins making up the cardiac muscle fiber, so it is an important indicator of MI-GOT is not specific to Myocardial Infraction, but it is also found in many acute diseases of liver and skeletal muscles which include heart muscle. Therefore it may be found in Myocardial Infraction and chest pain-LDH (Lactate Dehydrogenase) is not specific to cardiac muscle. It is found in liver and red cells. LDH is considered a secondary indicator of MI (Gaw, 1995; Marray, 1993). We collected required amount of data for diagnosis and divided it into two classes, one for learning and the other for testing. Finally we evaluated all the samples using the three approaches, namely SVM-linear classifier, SVM-nonlinear classifier and artificial neural networks (back propagation method).

\section{MATERIALS AND METHODS}

Neural networks: Neural networks are important learning machine techniques denoting a set of connectionist models inspired in the behavior of human 
brain. Artificial neural networks becomes one of the most commonly used tools in solving many problems in various application domains like classification, approximation, forecasting, pattern recognition and others. In practices the back propagation neural network is one of popular methods which provides a powerful linear, capable of nonlinear mapping (Luger, 2004). In recent years neural networks is used in medical diagnosis. In this study, neural networks has been used for classification and checking Myocardial-infraction. The designed neural network, has been trained by using Back propagation algorithm. The network topology is constructed to be feed forward: i.e., loop-free-generally connections are allowed from the input layer to the first, hidden layer; from the first hidden layer to the second, hidden layer to the output layer (Freeman and Skapura, 2003; Panzeri et al., 2001). Back Propagation networks (BP), is proposed for classification process. Each layer consists of a number of neurons which depends on the cases to be solved (Setiono, 2003). The proposed model shown in Fig. 1 is a three-layer feedforward NN that consists of 1-an input layer, consists of input features (measurement of labs. Testing for CK, GOT, LDH, Tr). 2-a hidden layer of 2 neurons. 3-an output layer of one neuron activated by logistic function, which determine if heart is healthy or not.

Support vector machines: The Support Vector Machine (SVMs) were first suggested from concepts of structural risk minimization, statistical learning theory and support vector classifier formulation (Vapnik, 1995).

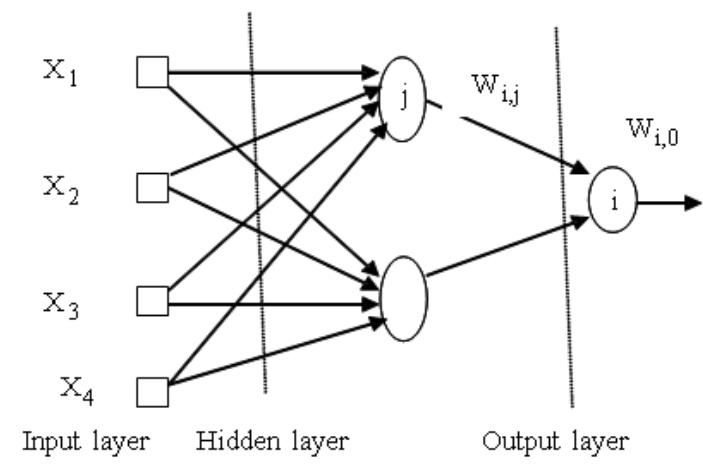

Fig. 1: Structure of feed forward neural networks
The Support Vector Machine (SVM) is a method for dividing a feature space using an optimized hyper plane, it means that SVM classifies data by determining a set of support vectors with different class labels that are members of the set of training inputs that outline a hyper plane in the feature space. The target is to minimize complexity and error of the classifier. The usefulness of his functionality is that SVMs are excellent for modeling space with a few dataset (Sebled and Bucklew, 2000). The simplest implementation of the support vector machine uses two classes $(+1,-1)$ (Herbich, 2002). Class with-1 denotes negative diagnosis and class with +1 denote positive diagnosis. For example if heart is healthy then class with- 1 is appear to represent negative diagnosis. If the feature space of these two classes is linearly separable, a hyper plane can be used to divide the data. but if the features space is not linearly separable, then the space can be projected into a higher dimensional metric space, known as the Hilbert space, since as the user must select one of a kernel function (linear, polynomial or radial) for the SVM during the training process that selects support vectors along the surface of this function (Frohlich et al., 2003). The goal is to find a dimension where the feature space will be linearly separable.

\section{RESULTS}

The correctly classified instances of the experiments are summarized in Table 2, which shows that nonlinear_SVMs is able to increase the specificity from $90 \%$ by use of Neural Networks up to $97 \%$, that because the Kernel function of SVM use feature space for classification instead of input space, moreover, the maximum margin used in hyperplane gives better margin for the class.

Table 1: Summary testing results of used methods

\begin{tabular}{lll}
\hline & Methods & \\
& $\begin{array}{l}\text { Nonlinear using redial } \\
\text { Kernal function }\end{array}$ & $\begin{array}{l}\text { Neural network (back } \\
\text { propagation algorithm) }\end{array}$ \\
\hline Testing criterion & $\begin{array}{l}\text { Correctly classified } \\
\text { instance (\%) }\end{array}$ & $\begin{array}{l}\text { Correctly classified } \\
\text { instance (\%) }\end{array}$ \\
Testing result & 97 & 90
\end{tabular}

Table 2: Results of linear and nonlinear SVM methods

\begin{tabular}{|c|c|c|c|c|}
\hline & \multicolumn{4}{|l|}{ Method } \\
\hline & Standard liner & Kernel liner & $\begin{array}{l}\text { Nonlinear polynomial } \\
\text { Kernel function }\end{array}$ & $\begin{array}{l}\text { Nonlinear using radial Kernel } \\
\text { function with } g=0.0001\end{array}$ \\
\hline Testing criterion & $\begin{array}{l}\text { Correctly classified } \\
\text { instances (\%) }\end{array}$ & $\begin{array}{l}\text { Correctly classified } \\
\text { instances (\%) }\end{array}$ & $\begin{array}{l}\text { Correctly classified } \\
\text { instances (\%) }\end{array}$ & $\begin{array}{l}\text { Correctly classified } \\
\text { instances (\%) }\end{array}$ \\
\hline Testing result & 57 & 96 & 95 & 97 \\
\hline
\end{tabular}




\section{DISCUSSION}

This is not conclusive evidence that the radial basis SVM is superior to the other methods, but it is suggestive. Whereas classification result by standard linear in Table 1 is not persuasive, may be because of the not big enough amounts of data.

\section{CONCLUSION}

In the research reported in this study, Support Vector machines and neural networks have been applied on the task of classifying Myocardial-infraction and the most accurate learning methods have been evaluated. The study shows that SVMs outperform ANNs, where SVM has the most stable correctly classified instances, especially for nonlinear classifier. Since we have looked at two classifiers for SVM, the first was linear classifier and the second nonlinear classifier using Kernel function. The result strongly suggests that nonlinear SVMs can aid in the diagnosis of Myocardial-infraction.

\section{REFERENCES}

Freeman, J.A. and D.M. Skapura, 2003. Neural Networks, Algorithm: Applications and Programming Techniques. Person Education, Delhi, India, ISBN: 9788178081083, pp: 401.

Frohlich. H., O. Chapelle and B. Scholkopf, 2003. Feature selection for support vector machines by means of genetic algorithms. Proceedings of the 15th IEEE International Conference on Tools with Artificial Intelligence, Nov. 3-5, IEEE Xplore Press, USA., pp: 142-148.

Gaw, A., 1995. Clinical Biochemistry. W.B. Saunders Company, Philadelphia, PA., ISBN: 0443064831, pp: 176.

Herbich, R., 2002. Learning Kernel Classifiers: Theory and algorithms. MIT Press, Cambridge, Mass. ISBN: 0-262-08306-X, pp: 364.
Joachims, T. and D.E. Chemintz, 1998. Text categorization with support vector machines. Proceedings of European Conference on Machine Learning, ECML'98, Springer Verlag, Heidelberg, pp: $137-142$.

Luger, G.F., 2004. Artificial Intelligence: Structures and Strategies for Complex Problems Solving. 5th Edn., Addison Wesley, London, ISBN: 10: 0321263189, pp: 928.

Marray, R.K., 1993. Harper's-Biochemistry. PrenticeHall International, London, ISBN: 0838536581, pp: 806.

Mitchell, T.M., 1997. Machine Learning. McGraw-Hill Science/Engineering/Mathematics, New York, ISBN: 0070428077, pp: 432.

Panzeri, S., E.T. Rolls, F. Battaylia and R. Lavis, 2001. Speed of feedforward processing in multiplayer networks of integrate and fire neurons. Elect. J. Comput. Neural Syst., 423-440.

Pontil, M. and A. Verri, 1998. Support vector machine for 3D objective recognition. IEEE Trans. Patt. Anal. Mach. Intell., 20: 637-346. DOI: 10.1109/ISSPA.2007.4555386

Sebled, D.J. and J.A. Bucklew, 2000. Support vector machine techniques for nonlinear equalization. IEEE Trans. Sign. Process., 48: 3217-3226. DOI: 10.1109/78.875477

Setiono, R., 2003. Techniques for Extracting Classification and Regression Rules from Artificial Neural Networks. In: Computational Intelligence: The Experts Speak, Fogel, D. and C. Robinson (Eds.). IEEE Press Wiley, USA., pp: 99-114.

Vapnik, V.N., 1995. The Nature of Statistical Learning Theory. Springer Verlage, New York, ISBN 10: 0443044813, pp: 188.

Yu, G.X., G. Ostruchov, A. Geist and N.F. Samatova, 2003. An SVM-based algorithm for identification of photosynthesis-specific genome features. Proceeding of the 2nd IEEE Computer Society Bioinformatics Conference, Aug. 11-14, IEEE Xplore Press, USA., pp: 235-243. DOI: 10.1109/CSB.2003.1227323 